\title{
Effect of Nozzle Confinement in the Axial Swirler
}

\author{
Roman Yusupov, Ivan Litvinov* , and Sergey Shtork \\ Kutateladze Institute of Thermophysics, Novosibirsk, Russia
}

\begin{abstract}
This work is devoted to the study of unsteady flow with the precessing vortex core (PVC) formed at the exit of a compact vane swirler with varying vanes angle and nozzles diameters. Amplitude-frequency characteristics of the PVC were obtained using two microphones. The modified Strouhal number dependence have showed a good generalization of the data for all nozzle diameters. The averaged and phase-averaged distributions of three components of velocity have been measured via the LDA system. The increasing the recirculation zone at increasing nozzle diameter for the swirl parameter $S_{g}=0.53$ and $R e=1.5 \cdot 10^{4}$ was detected. The degeneration of PVC was detected for all studied nozzle diameters $\mathrm{D}=30$, $40,50 \mathrm{~mm}$. In case of smallest diameter $\mathrm{D}=30 \mathrm{~mm}$ the PVC ceases to be periodic due to the absence of a recirculation zone. The three-dimensional structure of the PVC is reconstructed by the phase averaging method and visualized using the Q-criterion. Formation of the shifted recirculation zone, outer secondary vortex (OSV) and inner secondary vortex (ISV) is observed.
\end{abstract}

\section{Introduction}

Nowadays, swirling flows have a wide range of applications [1]. Despite this, there are many unexplored effects that are observed in swirling flows. One of the most interesting phenomenon for studying is the vortex decay with the precessing vortex core (PVC) formation. PVC is a source of powerful flow pulsations, which have a negative influence on the equipment and can lead to serious damages of the power equipment [2].

The effect of the vortex core precession has been studied rather tightly. In particular, the influence of the swirl intensity on the frequency characteristics of this phenomenon has been surveyed [3]. However, many researchers try to understand how the level of flow swirling and the geometric dimensions of the region behind the swirler affect the vortex breakdown with the formation of PVC. In addition, the real working characteristic of the swirling device has not been known, that hinders generalizing of characteristics for the swirl flows generated by swirlers with different geometries. This work is devoted to the study of the influence of geometry of the nozzle located downstream the swirler on structure of the flow with the PVC phenomenon.

\footnotetext{
* Corresponding author: litvinov@itp.nsc.ru
} 


\section{Experimental set-up}

The working section which is shown in the Fig. 1 was used to study the influence of the vanes inclination angle and the outlet diameter on a swirling jet structure. Air from a blower was supplied into the working section through the pipe with a diameter $d=40 \mathrm{~mm}$. The swirl of the flow was created by the vane swirler with a diameter $D_{0}=76 \mathrm{~mm}$. Then the swirling flow enters an open space through the nozzle. Vane swirlers with swirl parameters $S_{g}=0.53,0.94,1.37$ and nozzles with diameter $D=30,40,52 \mathrm{~mm}$ were used to vary the boundary conditions. The geometric swirl parameter was calculated using the formula, which is given in [4]:

$$
S_{g}=\frac{2}{3}\left(\left(1-\left(d_{*} / D_{0}\right)^{3}\right) /\left(1-\left(d_{*} / D_{0}\right)^{2}\right)\right) \cdot \operatorname{tg} \alpha
$$

where $D_{0}$ is swirler diameter, $d_{*}$ is diameter of central hub, $\alpha$ is angle of vane inclination.

LDA velocity data were recorded simultaneously with microphone signal to perform the phase average measurements. The microphone used to generate the reference signal of periodic vortex motion was a B\&K Type 2250 fitted with a probe tip as in [3]. A two component LDA «LAD 06» was used to acquire the velocity data.

Fig. 1. The experimental setup.

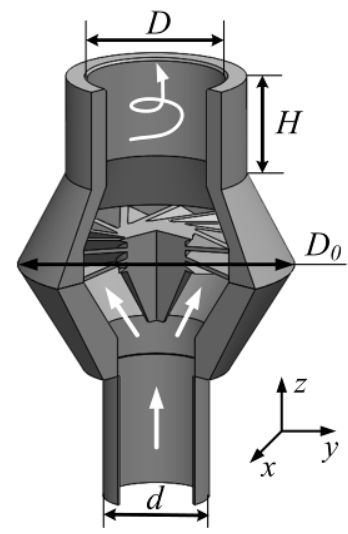

\section{Results}

\subsection{Frequency characteristics}

The frequency characteristics of the vortex core precession at the exit of the nozzle were measured using two microphones mounted in the opposite places flush with nozzle. Dominant frequency in pressure spectra was obtained by the FFT analysis of the difference signal recordings. The obtained dependences of the precession frequency on the air flow rate in a dimensionless form are shown in the Fig. 2. Dependencies are plotted for three values of the swirl number $S_{g}=0.53,0.94,1.37$ and for three nozzle diameters $D=30,40$, $52 \mathrm{~mm}$. The Reynolds numbers in experiments varied in the range from $10^{3}$ to $6 \cdot 10^{4}$.

It can be seen from the obtained dependences that at the nozzle diameter of 40 and 52 $\mathrm{mm}$ for the range of the investigated velocities the PVC effect has a self-similar behavior. Narrowing the exit region to $30 \mathrm{~mm}$ results in a weakening the vortex precession effect that becomes evident for smallest swirl number $S_{g}=0.53$ (Fig. 2a). Increase in the swirl number of the flow leads to the formation of a pronounced precession of the PVC with a subsequent 
increase in its frequency. Also Fig. 2c shows that for a high degree of swirl flow the data for different nozzle diameters fall on the same dependence.
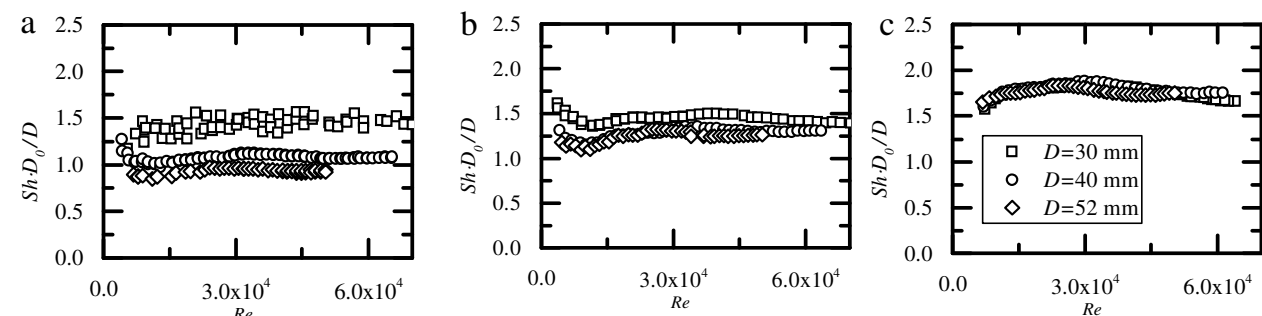

Fig. 2. Modified Strouhal number dependence of Reynolds number for three swirl cases and diameters of nozzle: $S_{g}=0.53$ (a), $S_{g}=0.94$ (b), and $S_{g}=1.37$ (c).

\subsection{Velocity distributions}

For a detailed understanding of the PVC behavior the measurements of the velocity field above the nozzle with a fixed value of the swirl parameter $S_{g}=0.53$ and for three nozzle diameters were performed using the LDA system. The obtained distributions of the averaged axial velocity component for a fixed Reynolds number $R e=1.5 \cdot 10^{4}$ are shown in Fig. 3. All data is nondimensioned by the diameter of the nozzle and the bulk velocity related to the nozzle.
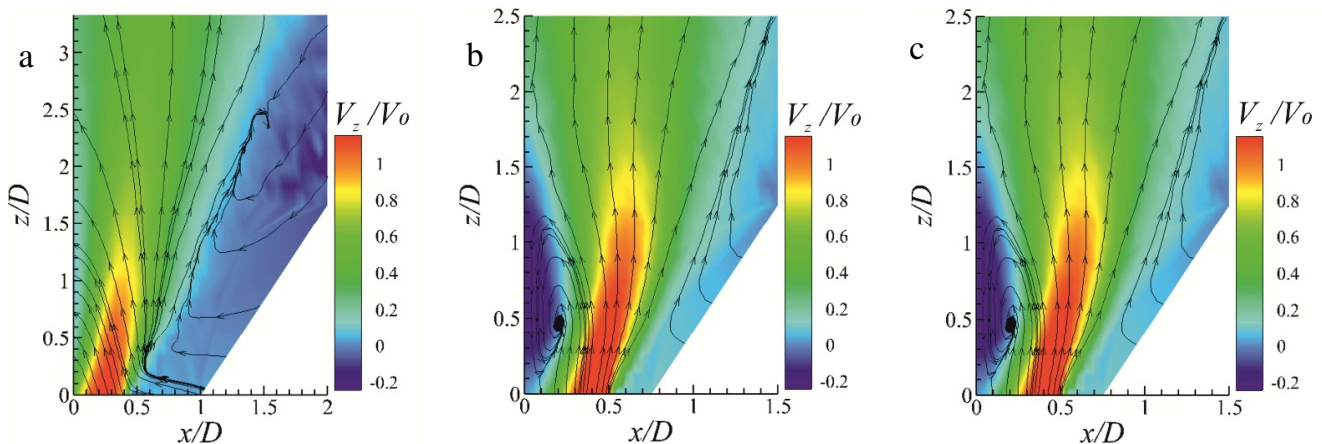

Fig. 3. The averaged axial velocity component at $S_{g}=0.53$ and $D=30 \mathrm{~mm}$ (a), $40 \mathrm{~mm}$ (b), $52 \mathrm{~mm}$ (c).

The time-averaged flow structure has shown the expected features of a swirling jet with a vortex breakdown and a central recirculation zone (RZ, area with negative axial velocity). Represented in Fig. 3a distribution of axial velocity over the nozzle with a diameter of 30 $\mathrm{mm}$ has shown the absence of reverse flow zone. Thus, the precessing vortex core is not distinct under the conditions of this experiment (Fig. 2a). Since, as noted in many works, the process of formation of the vortex precession is directly related to the formation of a recirculation zone along the axis of the swirling flow [5]. Increasing the diameter of the outlet nozzle gives rise to formation of an extensive reverse flow zone. In Fig. 3b, this region closes over the nozzle and in Fig. $3 \mathrm{c}$ it penetrates into the nozzle. From the presented distributions of the axial velocity component in Fig. 3 it follows that with an increase in the diameter of the output region, the zone of reverse flow increases in longitudinal and transverse dimensions.

\subsection{Phase-averaged flow map}

Fig. 4a presents a sample result on the phase-averaging of three components of velocity over an $x-y$ cross-section. It can be noticed that the RZ and the PVC are shifted away from the geometric centre. 
Fig. 4b presents the Q-criteria iso-surfaces obtained by the phase-averaged data processing with regard to periodic vortex motion associated with $S h \approx 1.75$. The iso-surface plotted with $\mathrm{Q}=0.05$ (positive), reveals two large-scale spiral vortices denoted here as the outer secondary vortex (OSV) and inner secondary vortex (ISV) as in the work [6].

a

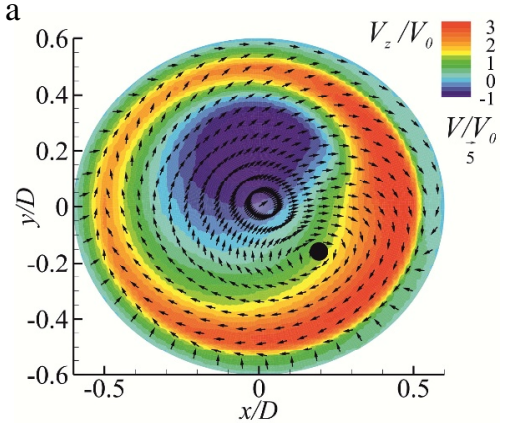

b

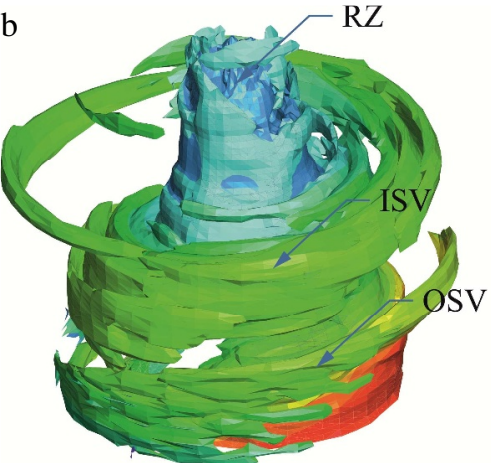

Fig. 4. Phase-averaged flow map $\left(S_{g}=1.37, D=52 \mathrm{~mm}\right)$ : velocity distribution in the cross-section near swirler nozzle $\mathrm{z}=3 \mathrm{~mm}$ (a), iso-surface of constant Q-criteria for visualization vortex structure in the axial swirler ( $V_{z}$ component of velocity pictured by color).

\section{Conclusions}

In this work the aerodynamic unsteady flow with formation of a precessing vortex core at the exit of the vane swirler has been investigated. The frequency characteristics of the PVC have been obtained using the microphone recording of the periodic pressure pulsations above the nozzle. The experimental data have showed a self-similar behavior of the PVC phenomenon with nozzles diameter of 40 and $52 \mathrm{~mm}$ and the variation of the swirl parameter. The flow regime at $S_{g}=0.53$ and $D=30 \mathrm{~mm}$ have showed the extinction of the PVC with non-periodic evolution in a swirling flow. For this case, the characteristic distribution of the averaged axial velocity component showed the absence of a recirculation zone. Further increasing in the diameter of the nozzle was accompanied by an increase of reverse flow zone. Using the phase-averaging method of the three velocity components, it has been found that the recirculation zone and the PVC are displaced relative to the geometric center of the flow. For reference, we have showed the reconstructed aerodynamic flow structure above the nozzle using the iso-surface of the Q-criterion, which showed the formation of two large-scale spiral vortices, outer secondary vortex (OSV) and inner secondary vortex (ISV).

The research was supported by Russian Science Foundation (Project № 18-79-00229).

\section{References}

1. S.V. Alekseenko, V.L. Okulov, Thermophysics and Aeromechanics 3, 2 (1996)

2. N. Syred, Progress in Energy and Combustion Science, 32 (2006)

3. I.V. Litvinov, S.I. Shtork, P.A. Kuibin, S.V. Alekseenko, K. Hanjalic, International Journal of Heat and Fluid Flow, 42 (2013)

4. K. Gupta, D. Lilley, N. Syred, Swirl Flows (Abacus Press, Kent, 1984)

5. T. Sarpkaya, J. Fluid Mech. 45, 3 (1971)

6. C.E. Cala, E.C. Fernandes, M.V. Heitor, S.I. Shtork, Exp. in Fluids, 40 (2006) 